\title{
Concepto de Área Natural Turística como aporte teórico para la planificación sustentable del Turismo
}

\author{
Juan Manuel Andrés y Matilde Elisabeth Encabo
}

RESUMEN

\begin{abstract}
El presente trabajo surge a partir de la investigación para la tesis de grado "El área natural y el turismo - Elaboración de un concepto de área natural como aporte para la planificación del turismo y la recreación en espacios naturales". Dado que el modo en que se entienda la realidad será determinante del tipo de planificación y accionar que se tenga sobre la misma, se ve la necesidad de resignificar el concepto de área natural turística. Contribuir a su entendimiento y a la búsqueda de un vocabulario homogéneo para los actores intervinientes, es de importancia para introducir racionalidad y coherencia al uso.

Para ello se definieron dos momentos metodológicos, uno de revisión documental, durante el cual se procedió a la búsqueda de información secundaria bibliográfica, y otro de revisión de experiencias a partir de entrevistas a informantes clave regionales y nacionales.

El resultado fue una redefinicion del Área Natural Turística (ANT) como un aporte conceptual a la planificación del turismo.
\end{abstract}

PALABRAS CLAVE

Área Natural Turística, Conservación, Planificación. 


\title{
The concept of Tourist Natural Area as theoretical contribution to sustaninable planning of Tourism
}

\author{
Juan Manuel Andrés y Matilde Elisabeth Encabo
}

\begin{abstract}
The present paper emerges from the research of the degree thesis "The natural area and tourism - Elaboration of a concept of natural area as a contribution to the planning of tourism and recreation in natural spaces".

Given that the way in which reality is understood will be determinant of the type of planning and actions to be taken on it, it is necessary to redefine the concept of tourist natural area. To contribute to its understanding and the search of a homogeneous vocabulary for the intervened actors is important to introduce rationality and coherence to the use.

For that, two methodological moments were defined; one of documental revision, during which the search of secondary bibliographical information was made, and another of revision of experiences extracted from interviews to regional and national key informants.

The result was a redefinition of Tourist Natural Area as a conceptual contribution to the planning of tourism.
\end{abstract}

\section{KEY WORDS}

Tourist Natural Area, Conservation, Planning. 


\section{FUNDAMENTACIÓN}

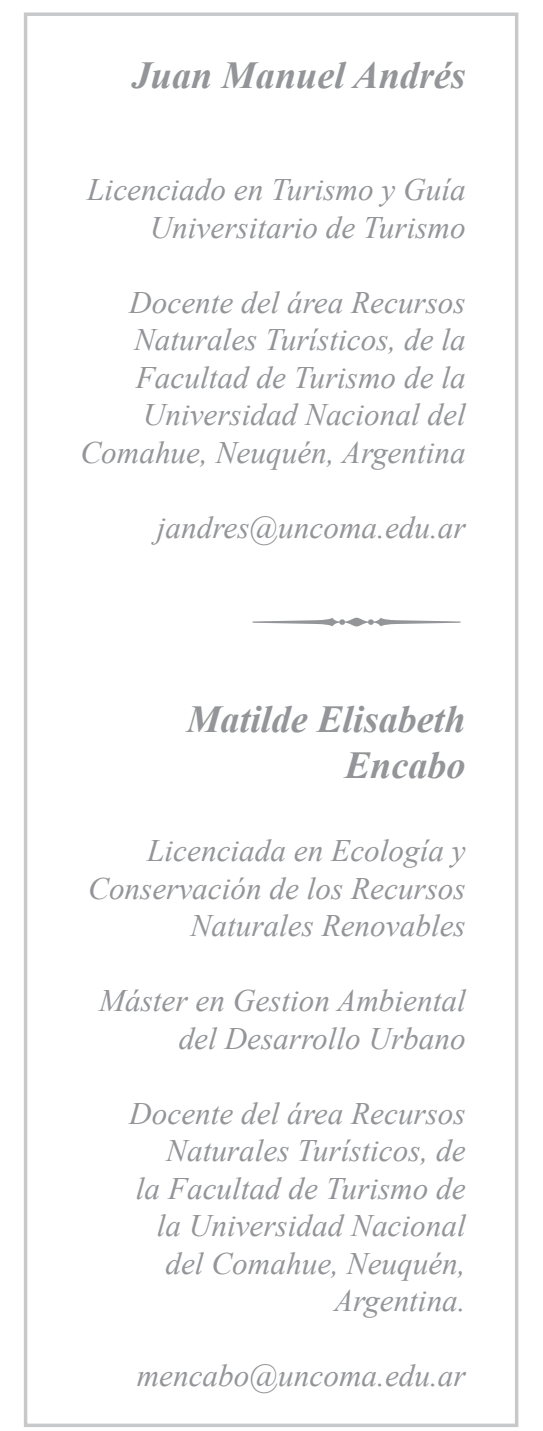

Históricamente las sociedades han debido acostumbrarse a una paradoja del conocimiento; por un lado, los individuos tienen una racionalidad acotada, por otro lado, y tal vez como causante de lo anterior, la realidad se muestra inabarcable, compleja y contradictoria (Zeballos de Sisto, 1992). Del conflicto que se establece entre este mundo complejo y ambiguo y la necesidad del hombre de entenderlo y de adaptarlo a sus esquemas mentales, surge la tendencia a simplificar y delimitar los diferentes aspectos del entorno. A los fines de posibilitar el análisis de los objetos en estudio, suelen resolverse situaciones problemáticas abstrayéndose de la totalidad e imponiendo límites arbitrarios, tomando en cuenta sólo ciertos factores (los más verificables), dejando fuera otros (García, 1991). Para intervenir en la realidad, una sociedad se ve en la necesidad de aproximarse a la misma desde el conocimiento (Galafassi, 2004). Sólo cuando se comprende, aunque sea esquemáticamente, la realidad en la cual se actúa; cuando se entiende el ambiente como contenedor de la vida y proveedor de los elementos necesarios para la reproducción de la sociedad, se está en posición de hacer uso de los recursos. Así, es posible afirmar que el modo en que se entienda la realidad será determinante del tipo de accionar que se tenga sobre la misma (Vigil, 1994). Por lo tanto, contribuir a ese entendimiento y a la búsqueda de un vocabulario homogéneo para los actores intervinientes, es de importancia para introducir racionalidad y coherencia al uso.

Cuando se planifica el turismo como una de las posibles actividades a desarrollar para lograr el crecimiento económico de una región, surge la necesidad por un lado, de conocer ese espacio de uso turístico y, por otro lado, de buscar / elaborar un modelo que permita comprender ese espacio como un todo, para optimizar la capacidad de uso de los recursos sin provocar cambios que impidan el uso actual y futuro. 


\section{ANTECEDENTES}

Los antecedentes del estudio del área natural turística deben ser buscados en las aproximaciones al espacio natural utilizadas por los planificadores de la actividad.

Históricamente han existido aproximaciones al espacio con una visión fundamentalmente práctica para el uso de los recursos naturales. Desde esta perspectiva, la necesidad de conocer la realidad busca aproximarse a la misma como sujetos neutrales y despojados de toda subjetividad. Es necesario contabilizar los atractivos, caracterizarlos y generar leyes para predecir todo comportamiento futuro. Esta forma de aproximarse al espacio natural, respondería al campo de las ideas positivistas, resultando un esfuerzo importante para sistematizar datos de los elementos naturales (recursos o atractivos) dando el puntapié inicial al estudio científico de los mismos.

Desde este punto de vista, el tratamiento de las áreas naturales turísticas para la planificación están referidos, en general, al relevamiento e inventariado de elementos naturales aislados, considerados como "atractivos". Se le brinda importancia a elementos naturales con unicidad y potencial suficiente para atraer flujos turísticos (Boullón, 1978). Este tipo de aproximación al inventario de recursos naturales se utiliza comúnmente en los planes de desarrollo turístico (Otero et al, 1999). La jerarquización de los atractivos se liga a las necesidades del mercado y a la posibilidad de obtener mayores flujos turísticos.

Desde mediados del siglo XX se comienza a generar, paralelamente a los avances logrados por el neopositivismo, una visión del espacio natural desde el naciente paradigma de la complejidad que considera sistemas complejos y dinámicos, en los cuales los recursos se encuentran interconectados, cumpliendo diferentes funciones (tangibles e intangibles) dentro de un sistema mayor.

La comprensión de la complejidad de los sistemas naturales y la inclusión de esta perspectiva dentro del análisis y conceptualización de las áreas naturales se transforma en una herramienta de importancia para lograr la sustentabilidad de las actividades turísticas. 
Algunos trabajos de investigación de finales del siglo XX consideran el área natural turística como un espacio de interrelaciones dinámico, un ecosistema donde existen conjuntos de atractivos interrelacionados en diferentes escalas, y donde cada escala de análisis podría ser considerada en sí misma como un atractivo a conservar para las generaciones futuras. Por caso, se ha trabajado a nivel de microcuencas turísticas en la cuenca Lácar del Parque Nacional Lanín, Argentina (Bergallo et al, 1997; 2000), donde incluso se ha contemplado las diferentes zonificaciones de la unidad de conservación para tomar en cuenta diferentes planificaciones de las ANT (Áreas Naturales Turísticas).

En cuanto a la concepción teórica del concepto de área natural, hasta la actualidad pocos autores han ahondado en el tema. Para Hatt, al área natural comprende "una serie de factores espaciales y sociales que actúan como influencias coactivas sobre todos los habitantes de un área geográfica" (Hatt, 1974).

Hawley entiende que el término área natural se utiliza muchas veces para hacer referencia a cualquier área físicamente delimitada por ríos, costas, montañas u otros rasgos semejantes, que constituyan una unidad fisiográfica definida. Este término suele aplicarse también a áreas de clima, tipo de suelo o vegetación uniformes, y a áreas que poseen una combinación de estos factores. El área natural puede ser definida como cualquier porción de la superficie terrestre cuyas condiciones físicas sean homogéneas (Hawley, 1975).

También se ha definido al área natural como el lugar físico o espacio geográfico donde se conservan elementos característicos y/o especies autóctonas del mismo (Bertonatti, 2003)

Desde el punto de vista turístico-recreativo el área natural toma de los autores tradicionales, la idea de una delimitación espacial concreta, definiendo los factores sociales como predominantes en la delimitación más que los aspectos naturales propiamente dichos y también se rescata la importancia de la planificación desde el punto de vista de la conservación de los recursos inmersos en ella. 


\section{METODOLOGÍA}

El trabajo realizado para alcanzar el objetivo de reelaborar el concepto como aporte para optimizar las intervenciones y planificaciones turísticas-recreativas, fue producto de una investigación de tipo documental y cualitativa. Una investigación documental "se focaliza, en la recopilación documental, libros u otras fuentes secundarias en los que el investigador procede a su tratamiento teórico, (...) su objeto de estudio trata sobre la teoría y lo que se pretende es profundizarla y proceder a través de la documentación al descubrimiento de elementos que puedan contribuir a nuevos aportes." (Mendicoa, 2003) Es posible afirmar que esta investigación posee un componente exploratorio, dado que pretende ganar familiaridad sobre un tema y crear una base sobre la cual realizar otras investigaciones más concluyentes y proponer la aplicación de otras técnicas de investigación. El proceso se estructuró en dos momentos, uno de revisión documental, durante el cual se procedió a la búsqueda de información secundaria bibliográfica. Otro momento fue de revisión de experiencias (Selltiz, 1980) mediante el uso de entrevistas con diferentes grados de estructuración. En este momento, se pretendió buscar aportes de informantes clave. Los informantes se seleccionaron de manera intencional en base a su trayectoria en el trabajo del turismo en ambientes patagónicos desde el aspecto académico y desde el punto de vista de la gestión. También se seleccionaron referentes a nivel nacional que trabajaran el tema espacial en general y con los recursos y áreas naturales en particular.

Se le prestó importancia en este momento a los aportes realizados por Boullón (1978) en lo referente al espacio turístico por entender que el mismo ha dejado una impronta en la planificación y la investigación espacial del turismo de Argentina y América Latina.

Teniendo en cuenta lo anterior, la principal técnica de tratamiento de los datos fue el análisis de contenido de información tanto secundaria (sobre todo bibliográfica) como primaria (experiencia de informantes clave). Lo que se pretendió con este análisis fue indagar sobre el tema de estudio en diferentes publicaciones y en los aportes de los profesionales para una mejor comprensión de las áreas naturales turísticas. 


\section{INTRODUCCIÓN}

El importante crecimiento que ha tenido la actividad turística y recreativa en la última década (OMT, 2006) se refleja en la cantidad de turistas que recorren la Argentina, en el mayor número de empresas relacionadas con la hospitalidad y los servicios y en una, cada vez más importante, presión sobre los espacios naturales (Boschi et al, 2005). Ante esta realidad adquiere principal relevancia el modo en que se actúa sobre estos espacios y el modo en que se interpreta los mismos. Por esto es importante realizar aportes teórico-metodológicos que ayuden a unificar criterios de aproximación al espacio natural para un uso conservativo del mismo.

El presente artículo pretende echar luz sobre el proceso de conceptualización del Área Natural Turística (ANT) como una base teórica importante que ayude a la planificación de los espacios naturales. En particular está pensado para espacios de alta naturalidad y donde priman ciudades de tamaño intermedio.

Se procura superar la idea de considerar el ANT como un espacio sin ningún tipo de intervención antrópica, sin presiones sociales, sin problemáticas (sin por ello dejar de incluir esos espacios prístinos) y circunscrito a una enumeración de atractivos naturales elegidos por el turista que determina una concurrencia al mismo a partir de determinadas distancias (Boullón, 1978).

Es así que se arriba a una nueva conceptualización del ANT a partir del análisis de aportes bibliográficos y empíricos desde diferentes perspectivas de la geografía, la ecología y el turismo y la posterior síntesis de los elementos principales en un concepto que consideramos superador y dinámico. 


\section{LA SUSTENTABILIDAD}

Lo sustentable en turismo, hace referencia a aquellas intervenciones y planificaciones que prevean acciones reparadoras y rectificadoras para mantener los impactos negativos al mínimo, buscando no romper el equilibrio del sistema y maximizando los beneficios. (Dachary, 2002)

Según la OMT "Las directrices para el desarrollo sostenible del turismo y las prácticas de gestión sostenible son aplicables a todas las formas de turismo en todos los tipos de destinos, incluidos el turismo de masas y los diversos segmentos turísticos. Los principios de sostenibilidad se refieren a los aspectos ambiental, económico y sociocultural del desarrollo turístico, habiéndose de establecer un equilibrio adecuado entre esas tres dimensiones para garantizar su sostenibilidad a largo plazo." Así, el turismo sustentable debería hacer un uso óptimo de los recursos ambientales, respetar los aspectos socioculturales de las comunidades anfitrionas y buscar que los beneficios económicos se distribuyan equitativamente en la población receptora (OMT, 2004).

Desde el punto de vista del área natural turística resulta importante el uso de todos los elementos del sistema con una visión de uso actual sin inhabilitar usos a futuro. 


\section{ESPACIO}

Olivier Dollfus entiende que "el espacio geográfico es un espacio percibido y sentido por los hombres, tanto en función de sus sistemas de pensamiento como de sus necesidades. (...) cada grupo humano tiene una percepción propia del espacio que ocupa y que le pertenece. El significado del espacio cambia según los individuos y sus funciones, y también según las épocas"(Dollfus, 1982)

Existe una influencia mutua entre el espacio y la sociedad, de modo que al transformar con sus actividades al espacio original, la sociedad se encontrará ante un nuevo espacio diferente y deberá adaptarse a las nuevas condiciones, se verá transformada en el tiempo.

El espacio es el soporte de las sociedades, es el proveedor de las materias primas y contenedor de los residuos.

Mediante las relaciones de producción y poder, el hombre modifica el espacio, plasma en él su organización social y, como contrapartida, resulta impactado (positiva o negativamente) por las nuevas características espaciales. 


\section{LOS ATRACTIVOS NATURALES COMO SISTEMAS}

Adaptando la concepción de Sistema Recurso Natural (Morello, 1982), los atractivos turísticos y recreativos naturales que el hombre utiliza en función de su avance cultural y sus necesidades no pueden considerarse como elementos aislados, son parte de un sistema mayor. Cuando la sociedad hace uso de un atractivo produce un impacto sobre el mismo o sobre alguno de los elementos que lo sustentan. Este concepto toma en cuenta que los elementos que se extraen de la naturaleza no se encuentran aislados, sino que son parte de un sistema dinámico y abierto.

No solo son atractivos los elementos de la naturaleza, sino también determinadas funciones de los sistemas naturales que son satisfactoras de necesidades humanas. 


\section{ÁREA NATURAL TURÍSTICA}

Área Natural: es un recorte espacial basado en la interpretación de una realidad. Esta subdimensión espacial es diferenciada por cierta homogeneidad interna o por límites de tipo físico, e incluye a la sociedad y sus manifestaciones.

Los límites del área natural son función de sus características intrínsecas y de aspectos relativos al uso social y las necesidades de planificación de una actividad social.

La característica o proceso que brinde homogeneidad, así como los límites del área natural, dependerán del centro de interés del grupo que la utilice y de la escala de análisis ligadas a los objetivos de desarrollo del área. La homogeneidad interna surge de una interpretación social del conjunto de procesos naturales o de la presencia de elementos naturales. El número, escala o densidad de los mismos es variable.

Los límites pueden darse tanto por la homogeneidad y/o elementos físicos de borde como por delimitaciones conceptuales, problemáticas a resolver y/o necesidades de planificación.

Por Área Natural Turística (ANT) se entiende a una dimensión de análisis del espacio cuya homogeneidad interna está dada principalmente por la presencia y continuidad de elementos y procesos de diferentes grados de naturalidad que adquieren valor como atractivos turísticos, representan el entorno sistémico necesario para la existencia de los atractivos y/o se convierten en un conjunto sistémico atractivo. Los límites de las mismas pueden tener relación con las problemáticas, usos y/o necesidades de planificación de la actividad turística-recreativa.

\section{Abstracción de la realidad}

El primer inconveniente que se plantea cuando se conceptualiza un aspecto de la realidad es creer que el concepto se transforma en la realidad misma, actuando en consecuencia. Un sistema no es una realidad, es sólo un medio de simplificar una realidad compleja con el fin de hacerla más accesible e "investigable". 
Del mismo modo, es importante aclarar que un área natural turística sólo puede ser individualizada desde la teoría. La misma, como todos los conceptos, es una definición teórico/conceptual de un aspecto de la realidad. Es una abstracción social o, si se quiere, una interpretación de un fenómeno real percibido, para intervenir en la actividad turística recreativa.

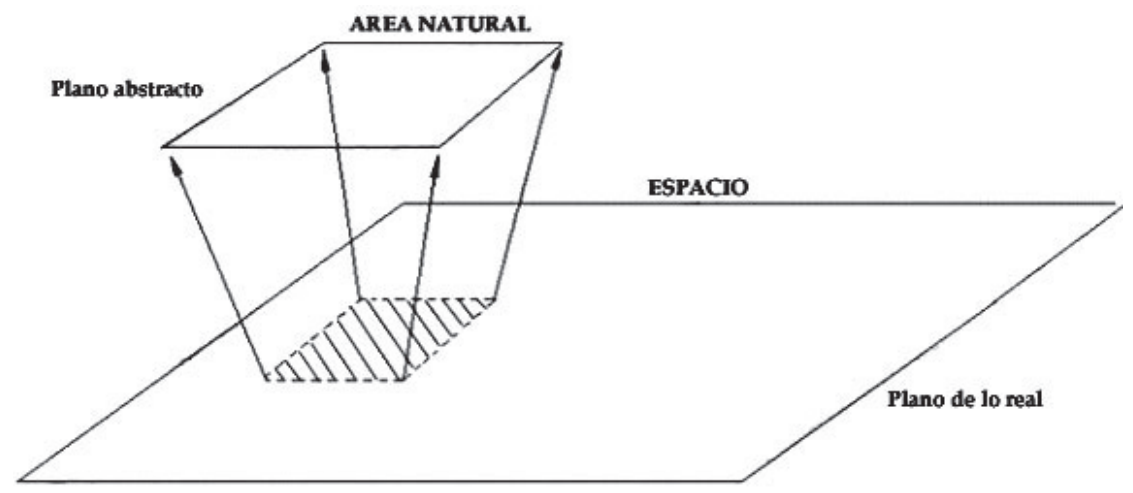

Fuente: Andrés \& Encabo 2007.

\section{Homogeneidad}

Para aislar o delimitar un sistema como parte de una realidad compleja, se suele tener en cuenta las relaciones de los elementos componentes o cierta homogeneidad perceptible que distinga a los elementos internos de los externos. Es decir que es necesario extrapolar la realidad de cada unidad a un conjunto.

En el caso de las Áreas Naturales Turísticas, es común que la homogeneidad percibida tenga que ver con las características intrínsecas del espacio considerado. Sin embargo, será necesario tener en cuenta también a factores externos que tienen que ver con los objetivos de investigación y/o planificación que un grupo social tenga sobre el área. Así, la homogeneidad interna (perceptible) será función del punto de focalización y la escala de análisis que se utilice. 
El punto de focalización se refiere a qué aspectos de la realidad serán relevantes en la investigación o planificación relacionados con los objetivos de la misma. Puede ser la flora, la fauna, los procesos que vinculan los elementos o los resultados de una actividad.

La escala de análisis es determinante en cuanto muestra con mayor o menor claridad al conjunto de elementos interactuantes. De tal modo que lo que en una escala es un elemento de un sistema, en otra es un sistema en sí mismo.

\section{Límites}

Los límites del área natural turística son, en una primera instancia, conceptuales. Desde el momento en que se conceptualiza el ANT, se están definiendo ciertos límites, se está acotando qué parte de la realidad puede ser considerada como área natural. Luego, los límites a establecer sobre el espacio pueden ser sugeridos por las características naturales del espacio, al observar barreras naturales (ríos, cadenas montañosas, cambios bruscos en biomas), o el alcance de la homogeneidad interna de cada sistema. El límite de un ANT estaría dado por la presencia de elementos distintos a los elegidos como determinantes de la homogeneidad interna.

Por último, los límites serán planteados desde afuera del sistema por el planificador o investigador, en base a sus objetivos.

\section{Espacio con algún grado de naturalidad}

Si se tiene en cuenta que la homogeneidad y los límites del ANT dependen de la mirada del investigador/planificador, y que los mismos varían en función de la escala de análisis y el punto de focalización, se puede decir que la "naturalidad" no se halla sólo en espacios prístinos. La homogeneidad de lo natural surge de una interpretación social del conjunto de procesos naturales o de la presencia de elementos naturales, pero el número, escala o densidad de los mismos es variable.

Así, se puede considerar un ANT al recorte de un espacio boscoso (natural o implantado), a un parque en una ciudad o a una laguna artificial. En todos los casos se incluye al hombre pensando 
y actuando en el sistema delimitado, como uno más de los componentes y como el principal modificador, así como éste incorpora, a través de valores culturales, a la naturaleza dentro del ámbito de lo social.

\section{Área natural turística como sistema de sistemas}

Un ANT es un sistema en el cual los subsistemas de atractivos interactúan. Desde esta perspectiva, no resultaría adecuado referirse sólo a atractivos aislados. Cada elemento se encontrará interconectado en mayor o menor medida con otros elementos y la importancia ya no deberá prestarse únicamente al atractivo, sino al sistema que lo sustenta. Las relaciones con los otros elementos de ese sistema originan las características que les dan valor como recurso turístico.

Entonces los atractivos del ANT pueden aislarse y a su vez la totalidad del sistema se transforma en un atractivo; no solo el elemento tiene valor sino el conjunto y sus funciones.

\section{Usuarios del Área Natural Turística}

Dado que el ANT es definida de acuerdo a objetivos sociales, que dentro de las mismas se incluye a la sociedad y que diferentes espacios pueden ser considerados ANT, entonces un espacio dentro de la ciudad o en el periurbano es ANT y los usuarios de las mismas pueden ser tanto turistas internacionales como recreacionistas y pobladores locales.

Por otro lado, los espacios con alta naturalidad también son utilizados por pobladores de centros urbanos cercanos.

Cualquier persona que visite un espacio (no importa el fin) es un usuario, que vivencia ese espacio, genera un impacto y tiene requerimientos según sus propios objetivos. El solapamiento de objetivos y actividades del conjunto de usuarios le da al ANT una alta complejidad.

A partir del análisis realizado sobre las dimensiones del ANT, se presentan cuadros comparativos entre el tratamiento que se ha dado hasta la actualidad y el que aporta esta nueva visión. 


\section{Homogeneidad del Área Natural Turística}

\begin{tabular}{|l|l|}
\hline \multicolumn{1}{|c|}{ Tratamiento actual } & \multicolumn{1}{c|}{ Nuevos aportes } \\
\hline $\begin{array}{l}\text { La Homogeneidad la definen los caracteres } \\
\text { naturales del espacio. Ejemplo: espacio de } \\
\text { bosque y de pradera. }\end{array}$ & $\begin{array}{l}\text { La homogeneidad depende de la escala, de } \\
\text { los objetivos de investigación, planificación, } \\
\text { intervención turística - recreativa. }\end{array}$ \\
\hline
\end{tabular}

Fuente: Andrés \& Encabo 2007.

\section{Límites del ANT}

\begin{tabular}{|l|l|}
\hline \multicolumn{1}{|c|}{ Tratamiento actual } & \multicolumn{1}{c|}{ Nuevos aportes } \\
\hline $\begin{array}{l}\text { Los límites están dados por las } \\
\text { características intrínsecas del espacio }\end{array}$ & $\begin{array}{l}\text { Los límites están dados por los objetivos de } \\
\text { la investigación, planificación, intervención } \\
\text { (ríos, montañas, Bosques) y en parte por el } \\
\text { manejo que se le realiza. }\end{array}$ \\
& \\
\hline
\end{tabular}

Fuente: Andrés \& Encabo 2007.

\section{Grado de Naturalidad del ANT}

\begin{tabular}{|c|c|}
\hline Tratamiento actual & Nuevos aportes \\
\hline $\begin{array}{l}\text { El ANT está definida por espacios con } \\
\text { naturaleza prístina o alto grado de } \\
\text { naturalidad, sin presencia o escasa } \\
\text { presencia del factor antrópicoEjemplos: } \\
\text { Áreas Naturales Protegidas; espacios } \\
\text { ribereños; Montañas; Bosques naturales. }\end{array}$ & $\begin{array}{l}\text { El ANT está definida por espacios donde } \\
\text { se pone en valor procesos naturales y/o } \\
\text { elementos naturales, con presencia del factor } \\
\text { antrópico en cualquier medida.Ejemplos: } \\
\text { Áreas Naturales Protegidas; plazas de una } \\
\text { ciudad; canales de riego; Bosques naturales } \\
\text { y/o implantados. }\end{array}$ \\
\hline
\end{tabular}

Fuente: Andrés \& Encabo 2007. 


\section{Usuarios del ANT}

\begin{tabular}{|l|l|}
\hline \multicolumn{1}{|c|}{ Tratamiento actual } & \multicolumn{1}{c|}{ Nuevos aportes } \\
\hline $\begin{array}{l}\text { Hace hincapié en los turistas que realizan } \\
\text { actividades en espacios naturales de alta } \\
\text { convocatoria, como centros de ski, parques } \\
\text { nacionales, etc. }\end{array}$ & $\begin{array}{l}\text { Pone en primer plano al visitante al espacio } \\
\text { sea este turista, recreacionista, poblador, } \\
\text { trabajadores. }\end{array}$ \\
\hline
\end{tabular}

Fuente: Andrés \& Encabo 2007.

\section{Elementos del ANT}

\begin{tabular}{|l|l|}
\hline \multicolumn{1}{|c|}{ Tratamiento actual } & \multicolumn{1}{c|}{ Nuevos aportes } \\
\hline $\begin{array}{l}\text { El ANT está definida por número de } \\
\text { atractivos con diferente "jerarquía", dada } \\
\text { por su convocatoria a turistas. Es mayor } \\
\text { su jerarquía si los turistas vienen de áreas } \\
\text { lejanas como por ejemplo: Europa. }\end{array}$ & $\begin{array}{l}\text { El ANT está definida por sus funciones en } \\
\text { el ecosistema en que se inserta. El atractivo } \\
\text { algunos elementos que pueden poseer } \\
\text { mayor poder de convocatoria. }\end{array}$ \\
\end{tabular}

Fuente: Andrés \& Encabo 2007.

\section{Planificación del Área Natural Turística}

\begin{tabular}{|l|l|}
\hline \multicolumn{1}{|c|}{ Tratamiento actual } & \multicolumn{1}{c|}{ Nuevos aportes } \\
\hline Concepción Positivista & Concepción compleja \\
\hline & \\
Los Recursos naturales son atractivos & $\begin{array}{l}\text { Los recursos naturales atractivos } \\
\text { inderrelacionados en un sistema que "es" } y a \\
\end{array}$ \\
& la vez contiene \\
\hline
\end{tabular}




\begin{tabular}{|l|l|}
\hline Hincapié en los aspectos observables & Hincapié en lo fisonómico y lo sistémico \\
\hline $\begin{array}{l}\text { El atractivo es el eje de la planificación en las } \\
\text { áreas naturales turísticas }\end{array}$ & $\begin{array}{l}\text { Las áreas naturales turísticas es el eje de la } \\
\text { planificación turística }\end{array}$ \\
\hline $\begin{array}{l}\text { Hincapié en los aspectos tangibles del } \\
\text { atractivo }\end{array}$ & $\begin{array}{l}\text { Hincapié en los aspectos tangibles e } \\
\text { intangibles del atractivo en el espacio }\end{array}$ \\
\hline $\begin{array}{l}\text { Sustentabilidad turística en referencia a cada } \\
\text { atractivo en particular }\end{array}$ & $\begin{array}{l}\text { Sustentabilidad turística basada en la } \\
\text { conservación del espacio natural que } \\
\text { contiene a los atractivos }\end{array}$ \\
\hline
\end{tabular}

Fuente: Andrés \& Encabo 2007. 


\section{CONCLUSIONES}

El Área Natural Turística, en tanto parte del espacio y dimensión de análisis del mismo, se define no sólo por las características intrínsecas del área, sino por la necesidad de planificar sobre el turismo. El área es un fragmento del espacio tanto para uso turístico como para otras actividades. Es para varios grupos sociales y para varias sociedades. Es por lo tanto necesario, tener en cuenta en la definición del ANT no sólo a los elementos y procesos naturales que dan homogeneidad y proveen atractivos, sino también a los individuos y grupos sociales que viven y usan ese área, intervengan estos en la actividad turística o no. Será importante considerar tanto al turista como al recreacionista, al poblador local, al prestador de servicios local y al planificador (todos con sus necesidades, objetivos e ideas previas).

En esta definición ya no es suficiente tener en cuenta la presencia y distribución territorial de atractivos y la presencia de una planta turística. Cada destino (actual o potencial) tiene una "vocación de uso" turístico recreativo, que puede atraer a un tipo específico de visitante. Es por esto, que ya no parecen adecuados o suficientes los sistemas de jerarquización de atractivos. El atractivo dependerá de la valoración que le otorgue una sociedad, y esta valoración se puede modificar en función de modas y tendencias locales o globales.

El área natural turística puede ser definida tanto por la homogeneidad interna de procesos y elementos naturales como por las problemáticas y predominio de actividades de esparcimiento -turismo y recreación-. Se considerará a los elementos ambientales, los procesos naturales y a parte de la sociedad haciendo un uso particular de esa área.

La importancia de la presente definición de área natural radica en que ayuda a establecer límites de estudio/alcance de las actuaciones en espacios naturales, buscando preservar las relaciones y procesos que permitan mantener la naturalidad y la integridad del sistema que sustenta los atractivos naturales. 


\section{REFERENCIAS BIBLIOGRÁFICAS}

- Bergallo, A; M. Encabo, S. Sánchez, G. Torre \& P. Martínez (1997). Estudio ambiental de un área protegida para el uso turístico recreativo sustentable. Caso cuenca Locar, PN Lanin. Facultad de Turismo. UNCo. Neuquén,

- Bergallo, A; M. Encabo, S. Sánchez, G. Torre \& P. Martínez (2000). Alternativas Metodológicas para la Gestión ambiental turística de un área Protegida. Facultad de Turismo. UNCo. Neuquén,

- Bertonatti, C. (2003). Glosario básico de términos ambientales. Fundación Vida Silvestre Argentina.

- Boullón, Roberto (1999). Metodología de inventario Turístico OEA - CICATUR. México.

- Buzai, Gustavo (1999). Geografía global. El paradigma geotecnológico y el espacio interdisciplinario en la interpretación del mundo. Lugar. Buenos Aires.

- Callizo Soneiro, J (1991). Aproximación a la geografía del turismo. Editorial Síntesis. Madrid.

- Codes de Palomo, Isabel (1993). Ecogeografía, la complejidad del medio ambiente. CEYNE. Buenos Aires.

- Dachary, C. A. y Arnaiz Burne, S. M. (2002) Globalización, turismo y Sustentabilidad. Ed. Universidad de Guadalajara. Puerto Vallarta. México.

- Dollfus, Olivier (1982). El espacio geográfico. Oikos-Tau. Barcelona.

- Encabo, M; G. Torre \& A. Boschi (2001). Management of Conservation in Spaces of Recreational Use in "Tourism in South America" Gui Santana Editor. The Haworth Press. New York- London - Oxford.

- Encabo, M \& S. Sánchez (1996)Impactos del Visitante en un sendero de interpretación. Parque Ncional Galápagos, Ecuador. En Estudios y Perspectivas en Turismo. Vol.5 (4). CIET. Buenos Aires.

- Galafassi, G (1993). La relación medio ambiente-sociedad: algunos elementos para la comprensión de su complejidad. Revista Paraguaya de Sociología, año 30, № 86.

- García, Rolando (1991). Conceptos básicos para el estudio de sistemas complejos. En "Los problemas del conocimiento y la perspectiva ambiental del desarrollo". Editorial Siglo XXI. España. 
- Hatt, Paul (1974). En Theodorson Estudios de ecología humana. Labor. Barcelona.

- Hawley, Amos (1975). Ecología Humana. Tecnos. Madrid.

- Matteucci, S. \& Buzai, G (1998). Sistemas ambientales complejos. Herramientas de análisis espacial. Eudeba. Buenos Aires.

- Mendicoa, Gloria (2003). Sobre Tesis y Tesistas. Espacio. Bs As.

- Morello, Jorge (1982). Manejo integrado de recursos naturales. Cuadernos del CIFCA, Centro Internacional de Formación en Ciencias Ambientales. Madrid.

- Morin, Edgar (1998). Introducción al pensamiento complejo. Gedisa. Barcelona.

- Otero, A et al (1999). Plan de desarrollo recreativo turístico zona norte. CFI/Unco/ Gobierno de la Provincia del Neuquén.

- Reboratti, Carlos (1999). Ambiente y Sociedad. Conceptos y Relaciones. Ariel. Buenos Aires.

- Selltiz, Wrightsman et al (1980). Métodos de investigación en las relaciones sociales. RIALP. Madrid.

- Vigil, Carlos (1994). Aproximación a la problemática ambiental, elementos para su análisis. Editorial Biblos. Buenos Aires.

- Zeballos de Sisto, M. C (1991). Sociedades humanas y equilibrio ecológico. Editorial Letra Buena. Buenos Aires.

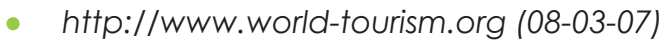

- http://www.metabase.net/docs/inguat/01036.html (28-05-2007)

- http://www.misiones.gov.ar/Concertacion/proyectos/PlanTuristico.htm (28-05-2007) 


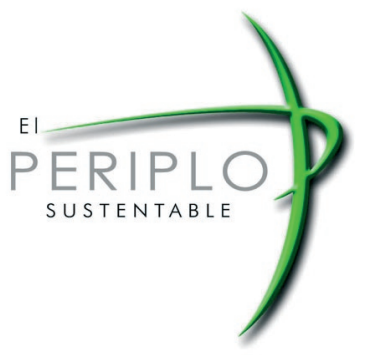

FICHA BIBLIOGRAFICA:

Andrés, J. M. y Encabo, M. E. Concepto de Área Natural Turística como aporte teórico para la planificación sustentable del Turismo

El Periplo Sustentable. Espacio de análisis y reflexión sobre Turismo Sustentable. México: Universidad Autónoma del Estado de México, enero de 2008, núm. 13

<http://www.uaemex.mx/plin/psus/rev13/articulo_04.pdfs.

[ISSN: 1870-9036]. 